\title{
Study on the Effect of Two Different Transfusion Methods in Neonates with Hyperbilirubinemia Induced by Non-Blood-Group Antibodies
}

\author{
Kunhai Wu' \\ Lufei Chen' \\ Huifang Huang ${ }^{2}$ \\ 'Blood Transfusion Department, Fujian \\ Provincial Maternity and Children's \\ Hospital, Fuzhou, Fujian, People's \\ Republic of China; ${ }^{2}$ Intensive Care Unit, \\ Fujian Provincial Maternity and Children's \\ Hospital, Fuzhou, Fujian, People's \\ Republic of China
}

\begin{abstract}
Purpose: The study aimed to analyse the clinical effect of homotype ET method and compatible ET method in neonates with hyperbilirubinemia caused by non-blood-group antibodies (non-blood-group antibodies refers to due to cause other than blood-group antibodies) as well as to provide relevant reference materials for clinical diagnosis and treatment in Fuzhou city.
\end{abstract}

Patients and Methods: Between January 2011 and December 2020, a total of 125 neonates, who were admitted to the Sentinel hospital of Fuzhou city, Fujian Provincial Maternity and Children's Hospital for hyperbilirubinemia and treated with ET therapy were selected. They were divided into homotype ET $(n=32)$ and compatibility ET $(n=93)$ treatment groups. We analysed changes in serum total bilirubin (STB), serum indirect bilirubin (SIB), and platelet count before and after ET; retrospectively analysed the neonates' clinical data; and compared findings between the 2 groups.

Results: The homotype ET group and the compatible ET group showed higher STB, SIB, and platelet levels before ET and decreased levels of the same after ET; the difference was statistically significant $(\mathrm{P}<0.001)$. Compared with the compatible ET group, the decreases in $\mathrm{STB}, \mathrm{SIB}$, and platelet count in the homotype ET group showed no significant difference $(\mathrm{P}>0.05)$.

Conclusion: ET therapy is the most timely and effective treatment method for severe hyperbilirubinemia in neonates clinically, and the effects of homotype and compatible ET are similar.

Keywords: hyperbilirubinemia, exchange transfusion, neonate, homotype, compatibility

\section{Introduction}

Severe neonatal hyperbilirubinemia $(\mathrm{SNH})$ is one of the most frequent reasons for hospitalisation or readmission in the first week of life worldwide. ${ }^{1}$ Neonatal jaundice is usually a physiologic condition and is one of the most common causes of hospital admissions in otherwise healthy new-borns. ${ }^{2,3}$ The main causes of neonatal hyperbilirubinemia include infection, enzyme deficiency, maternal and infant blood-group incompatibility, and unexplained hyperbilirubinemia. The pathophysiology of severe hyperbilirubinemia in new-borns is mainly due to the excessive lysis of red blood cells in the children's bodies, which produces a large amount of unconjugated bilirubin, causing damage to the functions of the body's various tissue systems. ${ }^{4}$ The function of various tissues and systems Will be damaged. Nuclear jaundice can damage the nervous system; severe disease will even kill or
Correspondence: Huifang Huang Intensive Care Unit, Fujian Provincial Maternity and Children's Hospital, Fuzhou, Fujian, People's Republic of China Tel +8613906913057

Fax $+86591-87555474$

Email190596715@qq.com 
cause disability. ${ }^{5,6}$ Exchange transfusion (ET) is a type of blood transfusion where the patient's blood or its components are exchanged with other blood or blood products, respectively. $^{7}$

ET is the most rapid and effective method for the clinical treatment of hyperbilirubinemia. The purpose of ET is to exchange large amounts of bilirubin, free antibodies, sensitised red blood cells, etc., an intervention, which enhances serum indirect bilirubin (SIB)-to-albumin binding. ${ }^{8}$

Thrombocytopenia is one of the most common adverse reactions of ET because the number of platelets decreases significantly after ET.

ET methods for neonates with hyperbilirubinemia caused by non-blood-group antibodies include homotype ET therapy and compatible ET therapy. At present, studies and analyses on the relative effects of these two ET methods are limited.

The aim of this study was to assess the relative effects of homotype ET therapy and compatible ET therapy for neonates with hyperbilirubinemia caused by non-bloodgroup antibodies.

\section{Patients and Methods Study Design}

This retrospective study was conducted on all neonates who underwent ET for hyperbilirubinemia caused by nonblood-group antibodies (use the hemolysis test of newborns) in the Sentinel hospital of Fuzhou city. Inclusion criteria were as follows: a. new-borns admitted to the hospital due to hyperbilirubinemia and treated with ET, b. meeting the eligibility standards for ET, ${ }^{9}$ and c. high bilirubin caused by non-blood-group antibodies-related blood disease.

We divided neonates into homotype ET and compatibility ET treatment groups; compared changes in serum total bilirubin (STB), SIB, and platelet count before and after ET; and retrospectively analysed the clinical data of the neonates and infants.

\section{Patients and Equipments}

Data on 252 neonatal cases of hyperbilirubinemia ET (2 cases of repeated ETaccounting for $0.79 \%$ were excluded) in the Department of Neonatology, Fujian Provincial Maternity and Children's Hospital, from January 2011 to December 2020 were collected, of which 125 met the inclusion criteria. They were divided into homotype ET $(n=32)$ and compatibility ET $(n=93)$ treatment groups.

The test instrument used for bilirubin was the Abbott al16200 (Abbott, Chicago, USA). The number of platelets was detected using the instrument Sysmex ${ }^{\circledR} \mathrm{XN}-3000$ (Sysmex Corporation, Kobe, Japan). The instrument used for blood type and neonatal haemolysis tests was the WADiana DG-57 (Diagnostic Grifols, Barcelona, Spain).

\section{Blood Selection and Dosage}

New-borns in homotype ET group with haemolytic disease caused by non-blood group antibodies received a homotype of blood composition similar to that of the child, and those who in compatibility ET group with haemolytic disease caused by non-blood group antibodies received a compatible of blood composition. Using the standard of $160-180 \mathrm{~mL} / \mathrm{kg}$ body weight, we strictly cross-matched blood, basing the amount of ET against the weight of the child.

ET was implemented through the simultaneous transfusion of peripheral arteriovenous double tubes. The scalp vein was selected as the ET route, as per routine, and the radial artery selected as the blood collection route. The total blood exchange volume was $160-180 \mathrm{~mL} / \mathrm{kg}$ body weight, and the difference between input and output blood volume generally did not exceed $3-5 \mathrm{~mL} / \mathrm{kg}$. The speed of ET was maintained at approximately $5 \mathrm{~mL} /(\mathrm{kg} . \mathrm{min})$, and the treatment time for ET was generally $1.5-2.0 \mathrm{~h}$. For every $100 \mathrm{~mL}$ of blood exchanged, $1 \mathrm{~mL}$ of $10 \%$ calcium gluconate was evenly supplied.

\section{Statistical Analysis}

All statistical analyses were performed using SPSS Statistics version 25.0 (IBM Corp., Armonk, N.Y., USA). The measurement data are expressed as mean \pm standard deviation (ie $\mathrm{X} \pm \mathrm{S}$ ), using the paired sample $t$-test and independent sample $t$-test, chi-squared test for the analysis of normative comparisons. P-values less than 0.05 were considered statistically significant.

\section{Results}

We identified 125 infants who underwent ET for hyperbilirubinemia caused by non-blood-group antibodies. Among the included cases, 82 were males and 43 females, the infants were more likely to be male $(65.6 \%)$, gestational age was 33-41 weeks, transfusion time ranged from 1 to 12 days, and birth weight was $2.3-3.9 \mathrm{~kg}$. They were divided into homotype ET $(n=32)$ and compatibility ET 
Table I Comparison of Gender, Gestational Age, Birthweight, STB, SIB, PLT and CA Before and After Homotype ET and Compatible ET Groups

\begin{tabular}{|c|c|c|c|}
\hline & $\begin{array}{c}\text { Homotype } \\
\text { ET }\end{array}$ & $\begin{array}{c}\text { Compatible } \\
\text { ET }\end{array}$ & $\mathbf{P}$ \\
\hline Gender & & & 1.000 \\
\hline Male & 18 & 64 & l \\
\hline Female & 14 & 29 & I \\
\hline $\begin{array}{l}\text { Gestational age } \\
\text { (weeks) }\end{array}$ & $38.37 \pm 1.518$ & $38.37 \pm 1.816$ & 0.711 \\
\hline Birthweight (kg) & $3.11 \pm 0.336$ & $3.11 \pm 0.496$ & 0.181 \\
\hline \multicolumn{4}{|l|}{ Before ET } \\
\hline STB I & $502.7 I \pm 8 I .598$ & $506.90 \pm 92.642$ & 0.821 \\
\hline SIB I & $463.22 \pm 75.802$ & $468.96 \pm 86.778$ & 0.740 \\
\hline PLT I & $271.97 \pm 98.896$ & $282.56 \pm 109.470$ & 0.630 \\
\hline CA I & $2.37 \pm 0.223$ & $2.33 \pm 0.256$ & 0.430 \\
\hline \multicolumn{4}{|l|}{ After ET } \\
\hline STB 2 & $262.93 \pm 73.604$ & $276.33 \pm 75.030$ & 0.383 \\
\hline SIB 2 & $240.07 \pm 68.36 \mathrm{I}$ & $259.37 \pm 66.210$ & 0.161 \\
\hline PLT 2 & $86.3 I \pm 42.97 \mid$ & $95.94 \pm 39.497$ & 0.247 \\
\hline CA 2 & $2.15 \pm 0.236$ & $2.14 \pm 0.269$ & 0.767 \\
\hline
\end{tabular}

Notes: $P<0.05$ indicates statistical significance, $P>0.05$ indicates no statistica significance. I: STB, SIB, PLT and CA before ET. 2: STB, SIB, PLT and CA after ET. Abbreviations: STB, serum total bilirubin; SIB, serum indirect bilirubin; PLT, platelet count; CA, calcium.

$(n=93)$ treatment groups. We can see that there is no difference in gender, Gestational age and birth weight between Homotype ET group and Compatible ET group, and there is no difference in STB, SIB PLT and CA levels between Homotype ET group and Compatible ET group before and after ET. These results are presented in Table 1.

Changes before and after Homotype ET were statistically significant $(\mathrm{P}<0.001)$. STB decreased from $502.71 \pm$ 81.598 to $262.93 \pm 73.604$, SIB from $463.22 \pm 75.802$ to $240.07 \pm 68.361$, the number of platelets from $271.97 \pm$ 98.896 to $86.31 \pm 42.971$ and $\mathrm{CA}$ from $2.37 \pm 0.223$ to $2.15 \pm 0.236$ (Table 2 and Figure 1).
Changes before and after compatible ET were statistically significant $(\mathrm{P}<0.001)$. STB decreased from $506.90 \pm$ 92.642 to $276.33 \pm 75.030$, SIB from $468.96 \pm 86.778$ to $259.37 \pm 66.210$, the number of platelets from $282.56 \pm$ 109.470 to $95.94 \pm 39.497$ and CA from $2.33 \pm 0.256$ to $2.14 \pm 0.269$ (Table 2 and Figure 2).

In the homotype ET group, STB, SIB, PLT and CA decreased by an average of $239.78 \pm 75.186,223.16 \pm$ $73.133,185.66 \pm 90.607$, and $0.22 \pm 0.162$ respectively. In the compatible ET group, STB, SIB, PLT and CA decreased by an average of $230.57 \pm 86.077,209.59 \pm$ 79.439 , and $186.62 \pm 101.320$, and $0.19 \pm 0.167$ respectively (Table 2 and Figure 3).

STB, SIB, PLT and CA in the homotype ET and compatible ET groups showed significant decreases; however, there was no significant difference in the effect of ET between the two groups.

\section{Discussion}

Neonatal jaundice is usually a physiologic condition and is one of the most common causes of hospitalisation. ${ }^{2}$ Generally speaking, in haemolytic disease of the newborn, hyperbilirubinemia appears early. It progresses rapidly and easily develops into bilirubin encephalopathy. ${ }^{10-12} \mathrm{SNH}$ is a major cause of neonatal morbidity and mortality, especially in low- and middleresource countries. $^{13}$

On one hand neonatal hyperbilirubinemia result when the new-born's liver is not fully developed and bilirubin fails to be excreted normally. On the other hand, the combined bilirubin is decomposed in the intestine, and the proportion of enterohepatic circulation is high. In the treatment of neonatal hyperbilirubinemia, the key to treating neonatal haemolysis is to promptly and effectively control the continued haemolysis and reduce the blood bilirubin concentration. More than half of the infants having severe hyperbilirubinemia underwent ET. ${ }^{14}$ Usually,

Table 2 Comparison of CA Before and After Homotype ET and Compatible ET Groups

\begin{tabular}{|l|c|c|c|}
\hline & Before ET & After ET & Average Difference \\
\hline Homotype ET & $2.37 \pm 0.223$ & $2.15 \pm 0.236$ & $0.22 \pm 0.162$ \\
Compatible ET & $2.33 \pm 0.256$ & $2.14 \pm 0.269$ & $0.19 \pm 0.167$ \\
t & 0.792 & 0.297 & 0.722 \\
P & $>0.05$ & $>0.05$ & $>0.05$ \\
\hline
\end{tabular}

Notes: $P<0.05$ indicates statistical significance, $P>0.05$ indicates no statistical significance.

Abbreviation: CA, calcium. 


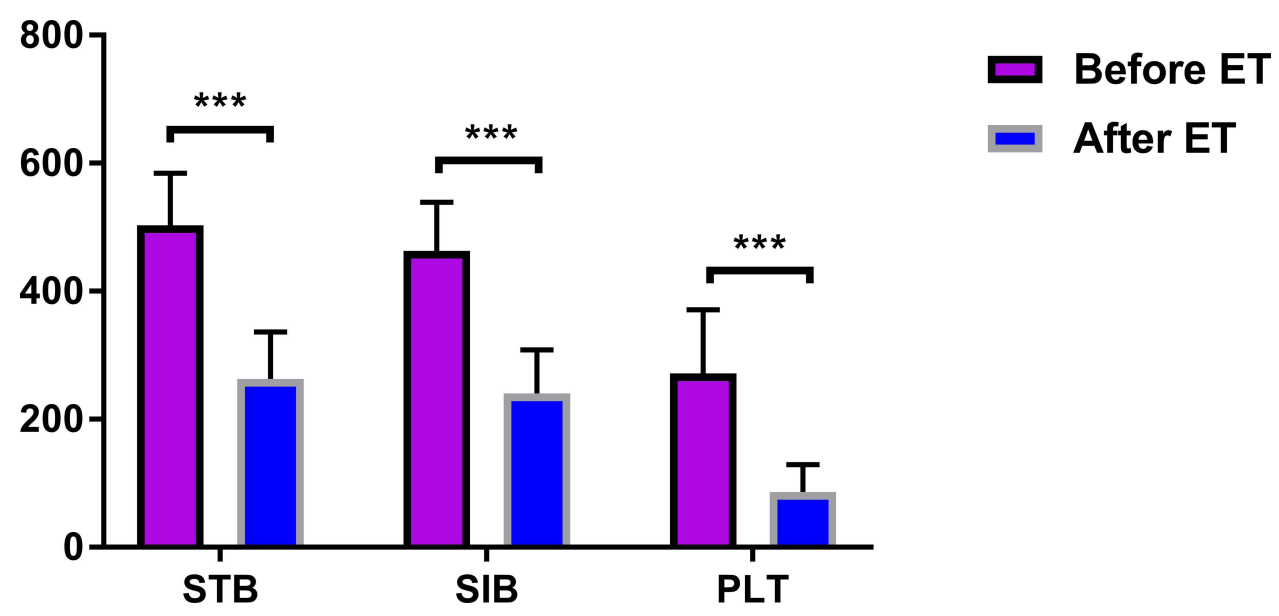

Figure I STB, SIB, and PLT before and after ET in the homotype ET group. ***P-values were considered statistically significant. Abbreviations: STB, serum total bilirubin; SIB, serum indirect bilirubin; PLT, platelet count.

the aim of ET is to remove red cells; however, since whole blood is drawn, white blood cells, platelets, and plasma cells are also removed. ${ }^{15}$

The study found that $33 \%$ of the children experienced a decrease in platelet count after ET. ${ }^{16}$ Some studies have reported that up to $54.6 \%$ of children undergoing ET therapy develop thrombocytopenia. ${ }^{17}$ One reason may be that the composition of blood transfused is related to that of the recipient. The blood we use is all-component blood with platelets removed, and the time of ET is relatively short. Thus, platelets in the blood circulation pool are quickly replaced, and the body temporarily fails to replenish platelets in time, resulting in a temporary decrease in the peripheral number of platelets. Hence, the clinician needs to pay close attention to the impact of ET on the number of platelets to avoid misdiagnosis and leakage diagnosis. At the same time, in view of the phenomenon of thrombocytopenia, platelet transfusion is performed when it is necessary to prevent the lethality of the bleeding of important organs. ET therapy has an impact on the number of platelets in the body; however, whether it affects the function of platelets requires further research. We have noticed that the children experienced a decrease in calcium after the ET. Clinical diagnosis and treatment should pay attention to prevent the appearance of hypocalcemia.

The degree of decrease in STB and SIB is the most important indicator of the effect of ET therapy. ET can prevent the occurrence of bilirubin encephalopathy. It not only reduces bilirubin concentration significantly in a short period of time but also exchanges immune antibodies and sensitised red blood cells in the body, reducing haemolysis. ${ }^{18}$ ET is of great significance in the treatment of neonatal

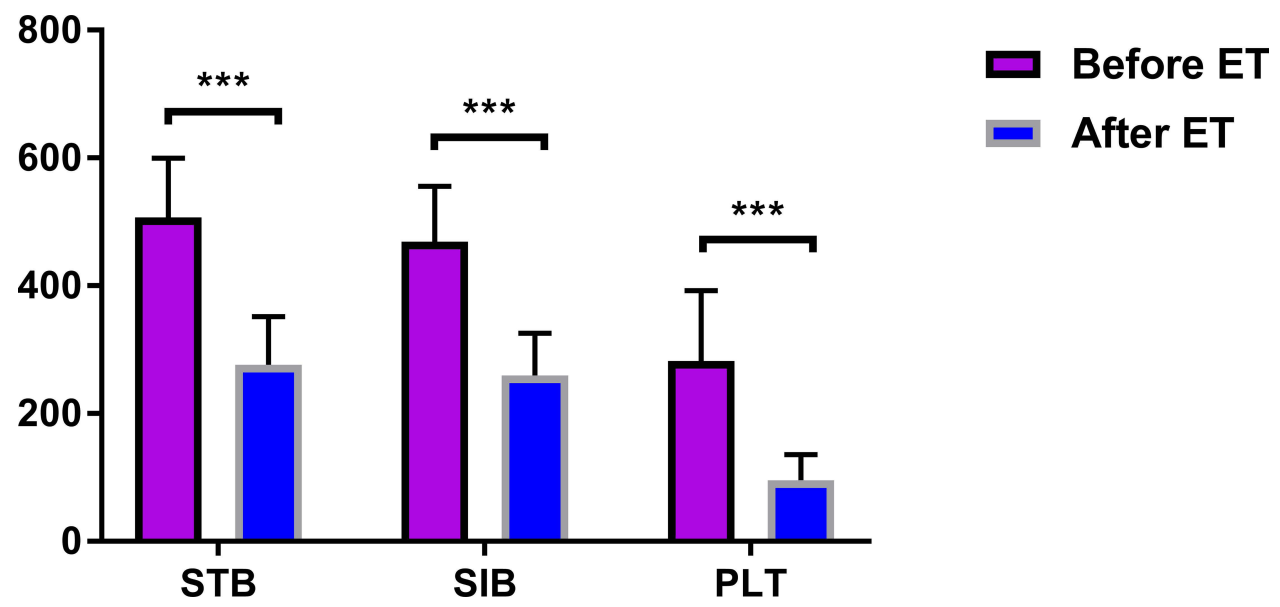

Figure 2 STB, SIB, and PLT before and after ET in the compatible ET group. ***P-values were considered statistically significant. Abbreviations: STB, serum total bilirubin; SIB, serum indirect bilirubin; PLT, platelet count. 


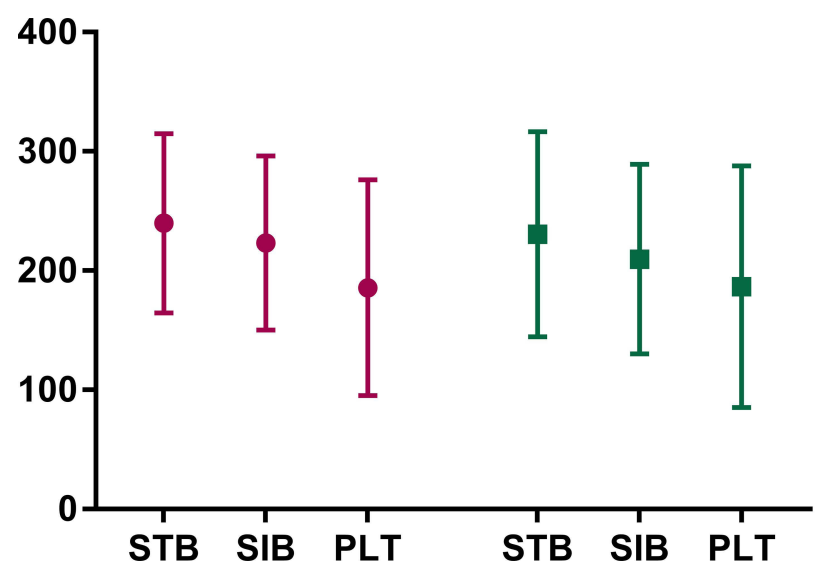

- Homotype ET

- Compatible ET

Figure 3 Average decrease in STB, SIB, and PLT in the homotype ET and compatible ET groups.

Notes: Average decrease in STB, SIB, and PLT in the homotype ET and compatible ET groups is not statistical significance. homotype ET : Average decrease in STB, SIB, and PLT in the compatible ET group.

Abbreviations: STB, serum total bilirubin; SIB, serum indirect bilirubin; PLT, platelet count.

hyperbilirubinemia. There are many causes of neonatal hyperbilirubinemia, of which blood-type incompatibility between the foetus and its mother is a common one. Studies have reported an association between ABO incompatibility and severe anaemia at birth. ${ }^{19,20}$ The more common ones are ABO-blood type or Rh-blood type incompatibility. In contemporary perinatal centres, $15 \%$ to $40 \%$ of neonates admitted for $\mathrm{Rh}$ or $\mathrm{ABO} \mathrm{HDN}$ require at least one $\mathrm{ET}^{21}$

The treatment of neonatal hyperbilirubinemia caused by blood-type incompatibility requires compatible ET. For neonatal hyperbilirubinemia caused by non-blood-group antibodies, either homotype ET or compatible ET is applicable. "Are both methods effective?" "Is there a difference between the two effects?" Research on this is presently limited.

Through the analysis of 125 cases of neonatal ET treatment of hyperbilirubinemia caused by non-blood group antibodies that were included in the study between January 2011 and December 2020 at our hospital, we discovered that the 32 cases in the homotype ET group and the 93 in the compatible ET group demonstrated significant differences in STB and SIB levels before and after ET, and that ET in general has a significant effect. This result is consistent with the results of previous research. ${ }^{22}$

Further analysis of the statistical difference between homotype ET and compatible ET showed that there was no difference in effectiveness between the homotype ET and compatible ET groups.

Although this study has obtained some information regarding the effect of ET on new-borns with severe hyperbilirubinemia caused by non-blood group antibodies, there are still some shortcomings. This study is limited to two time points, ie, before and after ET, and did not involve the changes in the observation indicators during ET. In addition, this study did not investigate the long-term effects of ET therapy on neonates.

\section{Conclusion}

In summary, the prevention and control of severe hyperbilirubinemia should be a priority, and various causes of its onset should be actively explored. ET can quickly and effectively treat neonatal hyperbilirubinemia, and it can save the lives of children at critical times. In Fuzhou city homotype ET and compatible ET are both effective for the treatment of severe hyperbilirubinemia caused by non-blood group antibodies. There is no difference in the effectiveness of the two methods for neonatal severe hyperbilirubinemia caused by nonblood group antibodies.

\section{Ethics}

This study was approved by the ethics committee of the Department of Gynecology of Fujian Provincial Maternity and Children's Hospital (the ethics approval number: 2021KR022). This article was a retrospective study, patient parental consent to review their medical records was not required by the ethics Committee of Fujian Provincial Maternity and Children's Hospital. This study strictly kept the patients' information confidential. The study complied with the Declaration of Helsinki. 


\section{Acknowledgments}

The authors would like to thank the Blood Transfusion Department, Fujian Provincial Maternity and Children's Hospital for helping to perform part of this work. We would like to thank Editage for English language editing.

\section{Disclosure}

The authors report no conflicts of interest in this work.

\section{References}

1. Bhutani VK, Zipursky A, Blencowe H, et al. Neonatal hyperbilirubinemia and Rhesus disease of the newborn: incidence and impairment estimates for 2010 at regional and global levels. Pediatr Res. 2013;74(1):86-100. doi:10.1038/pr.2013.208

2. Burke BL, Robbins JM, Bird TM, et al. Trends in hospitalizations for neonatal jaundice and kernicterus in the United States, 1988-2005. Pediatrics. 2009;123(2):524-532. doi:10.1542/peds.2007-2915

3. Battersby C, Michaelides S, Upton M, Rennie JM; Jaundice Working Group of the Atain. Term admissions to neonatal units in England: a role for transitional care? A retrospective cohort study. $\mathrm{Br}$ Med J Open. 2017;7(5):e016050.

4. Zhang BY. Analysis on the effect of blood exchange on neonatal hyperbilirubinemia. Nanning: Guangxi Medical University; 2012.

5. Song Y, Wang HW, Jiang CN, et al. Prognostic evaluation of neonatal hyperbilirubinemia by MRI. China Health Nutr. 2014;24:1199.

6. Liu LF. Clinical analysis of 68 cases of early neonatal severe hyperbilirubinemia. J Clin Exper Med. 2014;2:141-143.

7. Kim MS, Chung Y, Kim H, et al. Neonatal exchange transfusion: experience in Korea. Transfus Apher Sci. 2020;59(3):102730. doi:10.1016/j.transci.2020.102730

8. Muki S, Kumar P. Blood exchange transfusion for infants with severe neonatal hyperbilirubinemia. Semin Perinatol. 2011;35(3):175-184. doi:10.1053/j.semperi.2011.02.013

9. Editorial Board of Chinese Journal of Pediatrics, Neonatal Section, Chinese Medical Association of Pediatrics. Expert consensus on principles for diagnosis and treatment of neonatal jaundice. Chin $J$ Pediatr. 2010;48:685-686.

10. van Imhoff DE, Dijk PH, Hulzebos CV, et al. Uniform treatment thresholds for hyperbilirubinemia in preterm infants: background and synopsis of a national guideline. Early Hum Dev. 2011;87 (8):521-525. doi:10.1016/j.earlhumdev.2011.04.004
11. Maimburg RD, Olsen J, Sun Y. Neonatal hyperbilirubinemia and the risk of febrile seizures and childhood epilepsy. Epilepsy Res. 2016;124:67-72. doi:10.1016/j.eplepsyres.2016.05.004

12. Basu S, De D, Shukla RC, et al. Difference in cerebral blood flow velocity in neonates with and without hyperbilirubinemia. $J$ Epidemiol Glob Health. 2014;4(2):97-106. doi:10.1016/j. jegh.2013.09.008

13. Olusanya BO, Osibanjo FB, Slusher TM. Risk factors for severe neonatal hyperbilirubinemia in low and middle-income countries: a systematic review and meta-analysis. PLoS One. 2015;10(2): e0117229. doi:10.1371/journal.pone.0117229

14. Bozkurt Ö, Yücesoy E, Oğuz B, et al. Severe neonatal hyperbilirubinemia in the southeast region of Turkey. Turk J Med Sci. 2020;50 (1):103-109.

15. Amos RC, Jacob H, Leith W. Jaundice in newborn babies under 28 days: NICE guideline 2016 (CG98). Arch Dis Child Educ Pract Ed. 2017;102(4):207-209. doi:10.1136/archdischild-2016-311556

16. Al-Lawama M, Al-Rimawi E, Al-Shibi R, et al. Adoption of the American Academy of Pediatrics' neonatal hyperbilirubinemia guidelines and its effect on blood exchange transfusion rate in a tertiary care center in Amman, Jordan. J Blood Med. 2018;9:61-66. doi:10.2147/JBM.S162191

17. Yu C, Li H, Zhang Q, et al. Report about term infants with severe hyperbilirubinemia undergoing exchange transfusion in Southwestern China during an 11-year period, from 2001 to 2011. PLoS One. 2017;12(6):e0179550. doi:10.1371/journal.pone.0179550

18. Li HF. Clinical analysis of hyperbilirubinemia in neonates treated with exchange transfusion. Chongqing: Chongqing Medical University; 2013.

19. Gilja BK, Shah VP. Hydrops fetalis due to ABO incompatibility. Clin Pediatr. 1998;78(3):F220-1.

20. Drabik-Clary K, Reddy VVB, Benjamin WH, Boctor FN. Severe hemolytic disease of the newborn in a group B African-American infant delivered by a group O mother. Ann Clin Lab Sci. 2006;36 (2):205-207.

21. Smits-Wintjens VEHJ, Steggerda SJ, Oepkes D, et al. Bacillus cereus cerebral abscesses in a term neonate with Rhesus hemolytic disease treated with exchange transfusion. J Pediatr Infect Dis. 2010;5 (03):277-280. doi:10.3233/JPI-2010-0251

22. Liu Y. Feasibility study on treatment of neonatal severe hyperbilirubinemia with peripheral arteriovenous synchronous exchange of blood. Mod Drug Appl China. 2015;9:76-77.
International Journal of General Medicine

\section{Publish your work in this journal}

The International Journal of General Medicine is an international, peer-reviewed open-access journal that focuses on general and internal medicine, pathogenesis, epidemiology, diagnosis, monitoring and treatment protocols. The journal is characterized by the rapid reporting of reviews, original research and clinical studies across all disease areas. The manuscript management system is completely online and includes a very quick and fair peer-review system, which is all easy to use. Visit http://www.dovepress.com/ testimonials.php to read real quotes from published authors. 